Claremont Colleges

Scholarship@ Claremont

All HMC Faculty Publications and Research

HMC Faculty Scholarship

2-1-1993

\title{
Tissue Characterization and Imaging Using Photon Density Waves
}

Lars O. Svaasand

University of Trondheim

Bruce J. Tromberg

University of California - Irvine

Richard C. Haskell

Harvey Mudd College

Tsong-Tseh Tsay

Michael W. Berns

University of California - Irvine

\section{Recommended Citation}

Svaasand, L.O., B.J. Tromberg, R.C. Haskell, Tsay, T. and M.W. Berns. "Tissue characterization and imaging using photon density waves." Optical Engineering 32.2 (February 1993): 258-266. DOI: 10.1117/12.60749

This Article is brought to you for free and open access by the HMC Faculty Scholarship at Scholarship @ Claremont. It has been accepted for inclusion in All HMC Faculty Publications and Research by an authorized administrator of Scholarship @ Claremont. For more information, please contact scholarship@cuc.claremont.edu. 


\section{Tissue characterization and imaging using photon density waves}

\author{
Lars O. Svaasand, MEMBER SPIE \\ University of Trondheim \\ Norwegian Institute of Technology \\ Division of Physical Electronics \\ Trondheim N-7034, Norway \\ Bruce J. Tromberg \\ University of California at Irvine \\ Beckman Laser Institute and Medical Clinic \\ 1002 Health Sciences Road East \\ Irvine, California 92715-3054 \\ Richard C. Haskell, MEMBER SPIE \\ Harvey Mudd College \\ Physics Department \\ Claremont, California, 91711 \\ Tsong-Tseh Tsay \\ Michael W. Berns \\ University of California at Irvine \\ Beckman Laser Institute and Medical Clinic \\ 1002 Health Sciences Road East \\ Irvine, California 92715-3054
}

\begin{abstract}
The optical properties of brain tissues have been evaluated by measuring the phase velocity and attenuation of harmonically modulated light. The phase velocity for photon density waves at $650-\mathrm{nm}$ wavelength has been found to be in the range of 5 to $12 \%$ of the corresponding velocity in a nonscattering medium, and the optical penetration depth was in the range 2.9 to $5.2 \mathrm{~mm}$. These results are used to predict the resolution of optical imaging of deep tissue structures by diffusely propagating incoherent photons. The results indicate that structures of a few millimeters in linear dimension can be identified at $10 \mathrm{~mm}$ depth provided that proper wavelength and time resolution are selected. This depth can possibly be enlarged to $30 \mathrm{~mm}$ in the case of tissues with very low scattering such as in the case of the neonatal human brain.
\end{abstract}

Subject terms: biomedical optics; tissue optics; optical imaging; time-resolved techniques; frequency-resolved techniques.

Optical Engineering 32(2), 258-266 (February 1993).

\section{Introduction}

Optical techniques represent a valuable tool for in vivo analysis of tissue properties and for imaging of tissue structures. $^{1,2}$ Recent developments have emphasized dynamic measurements where either an ultrashort laser pulse or highfrequency amplitude-modulated laser light is launched into the tissue. ${ }^{3-8}$ The properties of the transmitted light range from quasi-coherent properties of the almost directly transmitted early part of the light to the almost randomized incoherent extensively scattered late part. This paper considers the advantages as well as the limitations of using diffusely propagating incoherently scattered light for evaluation of tissue properties and for imaging. $6,7,9$

Irridiation with harmonically modulated optical beams will initiate density waves of diffusely propagating photons. The velocity of these waves, which is strongly dependent on the modulation frequency, can typically vary from the velocity of light as an upper limit, down to about $5 \%$ of

Paper BM-056 received July 1, 1992; revised manuscript received Nov. 1, 1992; accepted for publication Nov. 1, 1992.

(C) 1993 Society of Photo-Optical Instrumentation Engineers. 0091-3286/93/\$2.00. this value in highly scattering tissues. We will also demonstrate that image resolution in the time domain can be significantly improved by receiving only the first part of the diffuse transmitted light and that the resolution can be further improved by using an optical wavelength that is optimally selected with respect to the scattering and absorption coefficient. ${ }^{3,10-12}$ This paper, however, will be limited to the diffusion approximation. The propagation of nonscattered or almost nonscattered photons can therefore not be considered within the framework of this theory. Diffusion theory only accounts for heavily scattered photons that propagate with a reduced effective velocity.

\section{Optical Properties of Tissues}

Biological media have because of their complicated structure very complex irregular optical properties. The optical inhomogeneities are due to differences in the ability for optical polarization as well as in the optical absorption. These differences exist between the cells and their surroundings as well as within each individual cell.

The propagation of light in tissue is strongly influenced by this mechanism; light is very efficiently scattered out of an incident collimated beam. The light will typically be 
scattered into an almost isotropic distribution within a few millimeters from the source. In some heavy scattering tissues, such as in the case of adult brain tissues, this randomization takes place within a fraction of a millimeter.

The total optical radiation in tissues is therefore composed of waves arriving from different directions with different optical phases. An important question is whether the phase relationships between these various waves are slowly varying or if they vary in a rapid and stochastic manner. The waves subjected to the first condition will add in a partially coherent manner. The total optical power will then be determined by the average value of the square of the total electric field. The power might then be higher or lower than the sum of the optical power in each wave, dependent on whether the waves add constructively or destructively. On the other hand, the waves with rapid and stochastic phase fluctuations will add incoherently. The total optical power will then always be the sum of the power of all participating waves. Light propagating in mammalian tissues will typically loose coherence after a few millimeters from the irradiated surface; the only exception to this rule is ocular media. The incoherent optical power can be characterized by the radiant energy fluence rate defined as the optical energy flux incident on an infinitesimal small sphere divided by the cross-sectional area of that sphere. ${ }^{13}$ This case is illustrated by Fig. 1. The photons are scattered in the tissue, and each cell will be irradiated with incoherent light coming from all directions.

\section{Propagation of Harmonic Waves}

The diffuse scattered optical energy will propagate to distal locations in tissue by a mechanism very much of the same nature as an ordinary diffusion process. The net transport of the diffuse photons will occur only from regions with a high fluence rate to regions with smaller values. The dynamics of this process can conveniently be described by expressing the fluence rate in terms of harmonic waves. These waves, which characterize the temporal and spatial variations in the photon density, can be expressed in the form, ${ }^{6}$

$\varphi(t, x) \propto \cos \left(\omega t-k_{i} x\right) \exp \left(-k_{r} x\right)$,

where $\varphi(t, x)$ is the value of the fluence rate at position $x$ in space at time $t$.

The waves are characterized by the angular frequency $\omega$, which gives the change in radians per unit time, and by the angular repetency $k_{i}$, which gives the change in radians per unit length in space. ${ }^{13}$ The relation between the angular frequency and the period in time, $T$, and the one between the angular repetency and the period in space, i.e., the wavelength $\lambda$, are

$\omega=2 \pi / T, \quad k_{i}=2 \pi / \lambda$.

The attenuation of the waves is given by the attenuation coefficient $k_{r}$. The reciprocal value of this coefficient is equal to the optical penetration depth, $\delta$, corresponding to a decay of the amplitude to $1 / e$ or $37 \%$ of the initial value.

The propagation of a photon density wave is shown in Fig. 2. The density of the photons is given by the amplitude of the solid curve, and the direction of the individual photons is illustrated by the arrows.

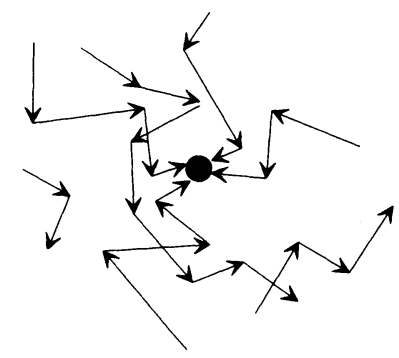

Fig. 1 Propagation of photons in scattering media.

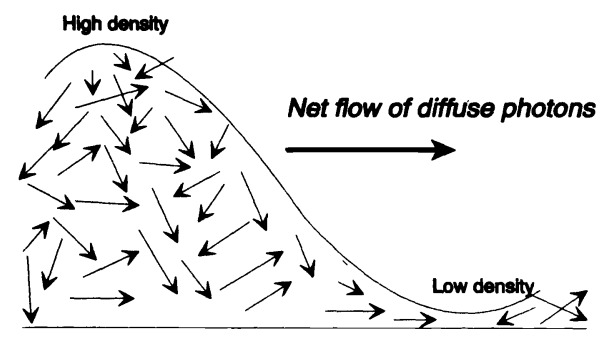

Fig. 2 Representation of photon density wave from high to low fluence rate regions.

\section{Propagation of Lossless Waves}

Because the photons flow from high- to low-density regions, the net transport of diffuse photons in the positive $x$-direction will require that the waves are attenuated. The flux of photons in an unattenuated wave during one period in time will result in the same amount in the backward direction as in the forward direction, and the net flux will be zero. The angular repetency $k_{i}$ and the attenuation coefficient $k_{r}$ are identically equal in any lossless diffusion process. They can be expressed in the form [see Eq. (13)]

$k_{i}=k_{r}=(\omega / 2 X)^{1 / 2}$,

where $X$ is the optical diffusivity. Optical diffusivity, in turn, is a function of the familiar optical properties described by the absorption $\beta$ and effective scattering $\sigma_{\text {eff }}$ coefficients [See Eq. (11)], which are, respectively, the reciprocal average distance between absorption and scattering events.

The diffusive waves are of the evanescent kind, and they are attenuated even in the absence of any loss mechanism. The total attenuation over a distance corresponding to one wavelength $\lambda$, since $\lambda=2 \pi / k_{i}=2 \pi / k_{r}$, is always equal to $\exp (-2 \pi)$. The amplitude of the waves is thus reduced to about $0.2 \%$ of the initial value over a distance corresponding to one wavelength. The waves also exhibit frequency dependent phase velocity. This velocity is given by [Eq. (13)]

$v_{\mathrm{ph}}=\frac{\omega}{k_{i}}=(2 X \omega)^{1 / 2}$.

Under lossless conditions, the phase velocity increase is proportional to the square root of the frequency. The phase velocity is infinitely large for waves of infinite high frequencies. This arises from the fact that the diffusion model is invalid for time scales shorter than the time between 
several scattering events. The propagation of those photons that only undergo a very limited amount of scattering must be described by the more complete electromagnetic equations.

The propagation of photons from a source can be characterized by a spectrum of harmonic diffusive waves. The high-frequency waves, since they have the highest velocity, will arrive first. But the high-frequency waves are the most heavily attenuated, and a noticeable increase in the photon density will occur only after arrival of waves with more moderate attenuation. This will occur when waves with reciprocal attenuation coefficients comparable to the distance arrive. The frequencies of these waves are given by [Eq. (3)]

$k_{r}=\left(\frac{\omega}{2 X}\right)^{1 / 2} \sim \frac{1}{L}$,

where $L$ is the distance. The order of magnitude for the time delay of arrival of these waves can therefore be expressed,

$t_{\text {delay }}=\frac{1}{\omega} \sim \frac{L^{2}}{X}$.

\section{Propagation of Waves with Loss}

The presence of photon absorption alters the properties of the waves in two significant ways. The absorption will primarily increase the attenuation. But this increased attentuation will, since a steepening of the density profile enhances the diffusion, secondarily result in an increased wave velocity. The absorption mechanism, which is important at all frequencies below the reciprocal optical absorption relaxation time (i.e., $\omega \tau \ll<1$ ) results in an attenuation and a velocity of the form [Eq. (13)]

$k_{r}=\frac{1}{(\tau X)^{1 / 2}}=\frac{1}{\delta}$,

$v_{\mathrm{ph}}=\frac{\omega}{k_{i}}=2\left(\frac{X}{\tau}\right)^{1 / 2}$,

where $\tau=1 / \beta c$ is the absorption relaxation time and $\delta=$ $\left[3 \beta\left(\beta+\sigma_{\text {eff }}\right)\right]^{-1 / 2}$ is the optical penetration depth.

The attenuation and the phase velocity are now frequency independent. This latter property has the important consequence that the velocity of the optical energy is frequency independent and equal to the phase velocity. The time delay for the envelope of an optical pulse to be transported over a distance $L$ is thus [Eq. (7)]

$t_{\text {delay }}=\frac{L}{2\left(\frac{X}{\tau}\right)^{1 / 2}}$.

The relations between frequency, angular wavenumber (repetency), and attentuation are shown in Fig. 3 [Eq. (13)]. The upper curve in Fig. 3 (series 2) shows the frequency in $\mathrm{GHz}(f=\omega / 2 \pi)$ versus the angular wavenumber in inverse meters (i.e., in radians/meter). This curve gives the dispersion relation between frequency and repetency in a region where the presence of loss is important. This curve

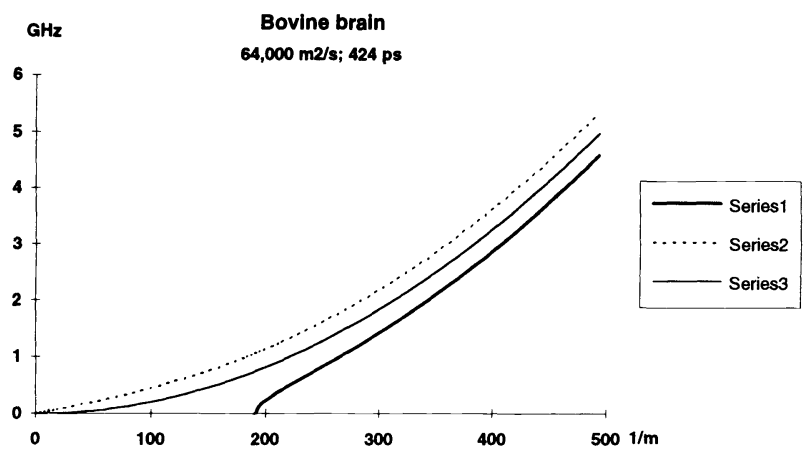

Fig. 3 Relations between frequency, angular wavenumber and attenuation. The optical diffusivity $\left(X=64,000 \mathrm{~m}^{2} / \mathrm{s}\right)$ and the relaxation time ( $\tau=424 \mathrm{ps}$ ) correspond to values for bovine brain measured ex vivo at 650-nm wavelength (See Table 1). Series 1, 2, and 3, respectively, correspond to attenuation $k_{r}$ with loss (lower curve), repetency $k_{i}$ with loss (upper curve), and the lossless case where $k_{r}=k_{i}$ (middle curve).

approaches, as follows from Eq. (13), the lossless case for high frequencies, i.e., for frequencies much higher than the reciprocal relaxation time $(f>>1 / 2 \pi \tau=0.38 \mathrm{GHz})$. In the low-frequency region, however, the curve approaches zero with a finite angle with the horizontal axis. This finite angle represents the nonzero phase velocity as given by $\mathrm{Eq}$. (7). The lower curve (series 1) gives, correspondingly, the relation between the frequency in $\mathrm{GHz}$ and the attenuation coefficient in inverse meters. The attenuation coefficient approaches the finite value of $k_{r}=1 / \delta$ in the zero frequency limit, whereas in accordance with Eq. (13), $k_{r}$ approaches the value for the lossless case in the high-frequency limit. The middle curve (series 3 ) corresponds to the lossless case where the attenuation coefficient $k_{r}$ and the angular repetency $k_{i}$ are equal. The phase velocity and the attentuation are in this case, as follows from Eqs. (3) and (4), both approaching zero in the zero frequency limit.

The results from measurements of low-frequency phase velocity and attenuation in various tissues are given in Table 1. These data were acquired using previously described frequency-domain photon migration methods. ${ }^{14}$ Briefly, the photon migration instrument is a modified multiharmonic Fourier transform phase and modulation fluorometer (SLM, 48000-medium high frequency, Champaign, Illinois). Light is provided by a water-cooled argon-ion laser (Coherent Innova 90-5) pumping a dye laser. A Pockels cell driven by the amplified output of a harmonic comb generator is used to produce light pulses with high harmonic content. The harmonic comb generator output, in the frequency domain, is a fundamental frequency (typically $5 \mathrm{MHz}$ ) and its integer harmonics at 5,10 , and $15 \mathrm{MHz}$, etc., to $250 \mathrm{MHz}$. Modulated light is coupled to a $600-\mu \mathrm{m}$-diam fused-silica fiber optic probe and directed onto the sample.

A second fused-silica fiber (same dimension) collects the scattered light and transmits it to the measurement photomultiplier tube (Hamamatsu R928). The gain of the photomultiplier tube is modulated by a second harmonic comb generator and driven at the frequency of the Pockels cell plus a small difference frequency (the cross-correlation frequency). The sample's phase and amplitude response at each modulation frequency is contained within the corresponding cross-correlation frequency. Using the multiharmonic tech- 
Table 1 Optical properties of tissues.

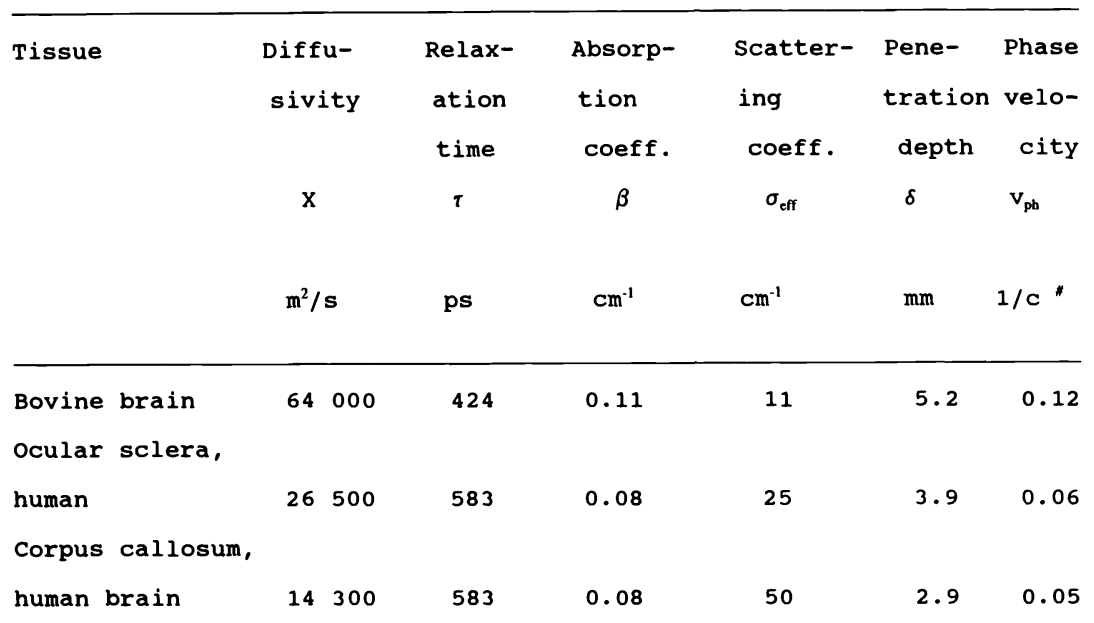

\footnotetext{
"Relative to velocity of light in a non-scattering medium with the same

index of refraction, i.e. $\mathrm{C}=2.14 * 10^{8} \mathrm{~m} / \mathrm{s}$, Wavelength $650 \mathrm{~nm}$, Measured exvivo
}

nique, we can acquire phase shifts and demodulations for 50 frequencies ( 5 to $250 \mathrm{MHz}$ ) in a few seconds. Brain data were recorded from fresh refrigerated samples under infinite medium conditions by inserting source and detector fibers into the material. Sclera data were collected under simulated infinite medium conditions by stacking several sections from fresh human globes. The resulting dimensions were approximately $15 \times 15 \mathrm{~mm}$, and boundary effects were minimized by immersing the entire sclera stack in $10 \%$ intralipid during measurements. Optical properties were determined from phase and amplitude information fit to a previously described expression [Eq. (13) and Ref. 7] for at least three source/detector separations. The results of Table 1 were found utilizing the technique and equipment as described in an earlier publication. ${ }^{14}$

Table 1 gives six different optical tissue parameters for each tissue. There are, however, only three independent sets of parameters. When the index of refraction is given, the optical properties can be characterized either by the optical diffusivity and relaxation time $(X, \tau)$, by the effective scattering and absorption coefficient $\left(\sigma_{\text {eff }}, \beta\right)$, or finally by the optical penetration depth and low-frequency phase velocity $\left(\delta, v_{\mathrm{ph}}\right)$. The relations between the optical diffusivity, relaxation time, effective scattering coefficient, and absorption coefficient are given in Eq. (13). The corresponding relations between the optical diffusivity, relation time, penetration depth $\delta$, i.e., the attenuation coefficient in the zero frequency limit, and phase velocity $v_{\mathrm{ph}}$, i.e., the phase velocity in the zero frequency limit, are given in Eq. (7). The data given in Table 1 range from tissues with more moderate scattering, such as in the case of bovine and porcine brain, to intense scattering media, such as the corpus callosum of the human brain.

\section{Propagation of Pulsed Optical Power}

The propagation of an optical pulse can be represented with a spectrum of harmonic waves, and the high-frequency con- tent of this spectrum increases with the shortening of the pulse. An infinitesimally short pulse releasing a finite amount of optical energy, i.e., a so-called Dirac or delta pulse, corresponds to an infinitely broad frequency spectrum of harmonic waves. The propagation of such a pulse is illustrated in Fig. 4.

An initial pulse of diffuse photons as shown on the left side in Fig. 4, broadens during propagation as illustrated by the pulse on the right side. The pulse broadening in a diffusion process occurs because the various frequency components of the pulse propagate with different velocities; the high-frequency components generally arrive before those with low-frequency. The time delay for the pulse envelope is also dependent on whether the rather lossless high-frequency waves or the more lossy low-frequency waves are the predominant ones in the broadened pulse. If the high-frequency components are the relevant ones, the delay is approximately given by $t_{\text {delay }}=L^{2} / X$, where $L$ is the traveled distance [Eq. (6)]. The corresponding time delay for the pulse envelope in the low-frequency case is given by $t_{\text {delay }}=L / v_{\mathrm{ph}}$ [Eq. (8)]. These two mechanisms are of equal importance when

$$
\frac{L^{2}}{X} \sim \frac{L}{2\left(\frac{X}{\tau}\right)^{1 / 2}}
$$

or

$L \sim \frac{1}{2}(X \tau)^{1 / 2} \sim(X \tau)^{1 / 2}=\delta$

The delay time for the arrival of the pulse envelope thus increases quadratically with distance until the pulse has traveled a length approximately equal to the optical penetration 


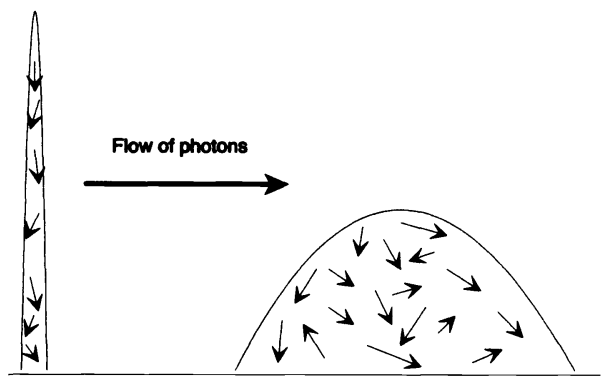

Fig. 4 Representation of pulse broadening during propagation.

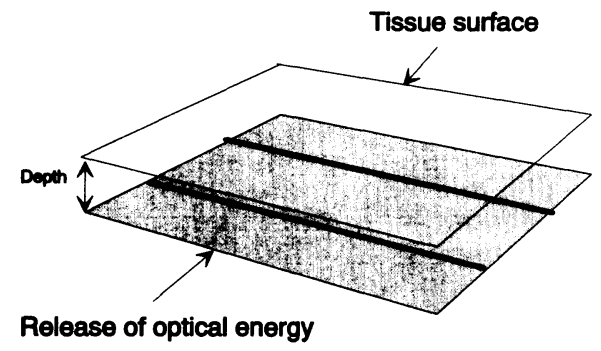

Fig. 5 Initial conditions for Fig. 6 simultations: release of optical energy below tissue surface.

depth. In the case of larger lengths, the delay time increases approximately linearly with traveled distance. The different properties of the various frequency components of an optical pulse have important implications in imaging of tissue structures. This is demonstrated by the series of simulations illustrated in Figs. 5 and 6. Figure 5 describes the model boundary conditions: two parallel absorbing lines, $1 \mathrm{~mm}$ in width, $10 \mathrm{~mm}$ apart, embedded in tissue at a depth of either 5 or $10 \mathrm{~mm}$ below the surface. A Dirac pulse is released below the surface at time $t=0$ in the absorber plane. Infinitely absorbing lines are selected to optimize contrast. Contrast enhancement in real imaging applications would, of course, involve some degree of optical frequency manipulation. In addition, we have necessarily selected relatively simple conditions that do not violate our diffusion theory framework. Thus, the Fig. 6 simulation involves observation of the shadow cast by each line under uniform field illumination. Real systems would employ more complex single- or multiple-point sources at a partially transmitting surface. These source and boundary conditions were intentionally imposed, however, to reveal more clearly the interplay between photon diffusion and spatial contrast.

Figure 6 shows the time development of the fluence rate for various conditions. The fluence rate was calculated from the diffusion equation with boundary solutions for a totally reflecting surface as derived in Sec. 8. The explicit solution for this fluence rate is given by Eq. (23). The vertical axis gives the surface fluence rate in $\mathrm{W} / \mathrm{m}^{2}$. As illustrated in Fig. 5, the optical energy is released in a horizontal layer below the surface. Here, the two black lines intersect at right angles, the marks in the middle of the $z$ axis. The second horizontal axis records time elapsed (picoseconds) after release of a Dirac pulse of $10^{4} \mathrm{~J} / \mathrm{m}^{2}$, roughly the maximum energy density that can be released without introducing thermal damage to the tissue.
The case of 5-mm-deep lines is illustrated in Fig. 6(a). The time axis spans from the release of the pulse to the arrival of the pulse envelope [Eq. (8)]. This delay is for bovine brain tissue equal to $t_{\text {delay }}=203 \mathrm{ps}$ for $L=5 \mathrm{~mm}$. The presence of the two individual lines is now clearly identifiable in the peak of the pulse. The corresponding results for 10-mm-deep lines are shown in Fig. 6(b). Here, the two individual lines are difficult to identify, but the general presence of the absorbing lines is still clearly visible. However, this information is almost completely lost on the tail of the pulse arriving after 814 ps [see Fig. 6(c)]. Clearly, much better resolution is possible if light is collected before the scattering has become too extensive. This can, in principle, be obtained by two different techniques. The first, and rather obvious one, is to receive only the first part of the pulse and reject all light that arrives later. The flank of the pulse arriving after 129 ps contains, as shown in Fig. 6(d), well-defined information on the presence of the two individual black lines. The second method is to shorten the delay of the pulse itself by selecting an optical wavelength with higher loss. As discussed previously, enhancement of the loss will increase the velocity of the pulse envelope. The propagation of a pulse at a wavelength corresponding to $10 \times$ higher loss is shown in Fig. 6(e). Under these conditions a pulse delayed only 129 ps contains information regarding individual lines at the peak of the pulse. This technique, of course, has the drawback that the flank of the pulse is somewhat attenuated.

The amount of this loss follows from Figs. 6(d) and 6(e); the maximum value of the fluence rate in Fig. 6(d) is a factor of about 15 larger than the maximum value in Fig. 6(e). The impact of reduced loss is demonstrated by Fig. 6(f). This figure shows the pulse after propagation $10 \mathrm{~mm}$ through a medium with the same optical diffusivity as the bovine brain, but with a loss corresponding to the ultimate minimum loss for tissues, i.e., that of sea water. The pulse in the sea waterlike phantom tissue, which has an optical relaxation time of $10 \mathrm{~ns}$ and an optical penetration depth of $\delta=25 \mathrm{~mm}$, will be dominated by the high-frequency waves. The time delay is therefore only dependent on the diffusivity, and the time delay is about $t_{\text {delay }}=1563$ ps as given by Eq. (6). The contrast is now very poor, and the presence of the absorbing lines is barely seen in the pulse amplitude.

These results, however, also reveal the limitations of the diffusion approximation. No upper limit exists for the wave velocity within this approximation; waves of infinite high frequencies have infinite high phase velocity. On the other hand, the attenuation of the high-frequency waves are heavily attenuated; waves of infinite high frequencies are infinitely heavily attenuated. This phenomenon is responsible for the nonzero values of the fluence rate at times shorter than the time of flight of nonscattered photons, i.e., $L / c$ $=47$ ps for Figs. 6(b) through 6(f). The diffusion theory based on a multiple scattering process is basically invalid for time scales comparable or shorter than the time of flight of nonscattered photons.

\section{Conclusions}

The properties of brain tissues reveal that optical imaging of tissue structures is also possible within the framework of diffusely propagating incoherent photons. However, the depth of resolution for time-independent imaging is very limited 
Bovine brain, 650nm (64,000m2/s424ps) Lines @ $5 \mathrm{~mm}$ depth (0-203ps)

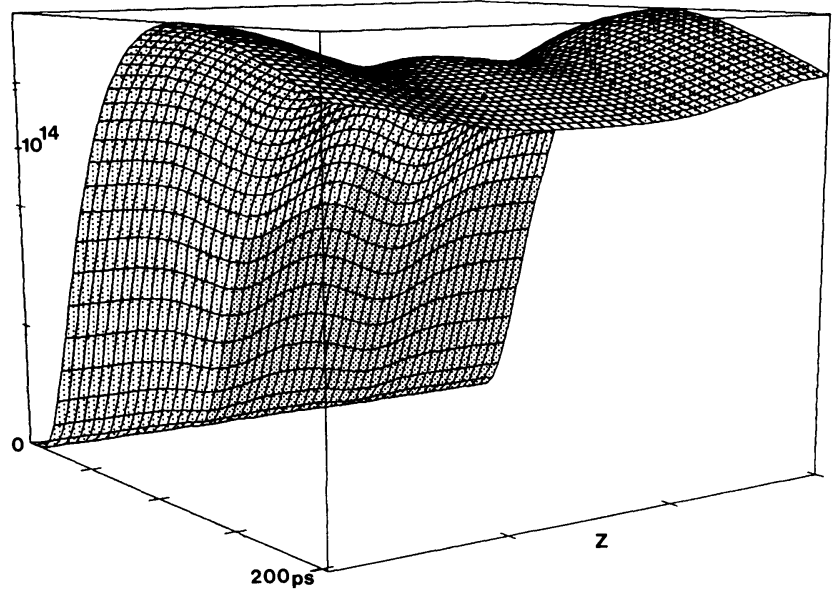

(a)

Bovine brain, $650 \mathrm{~nm}(64,000 \mathrm{~m} 2 / \mathrm{s}, 224 \mathrm{ps})$ Lines @ $10 \mathrm{~mm}$ depth (0-814ps)

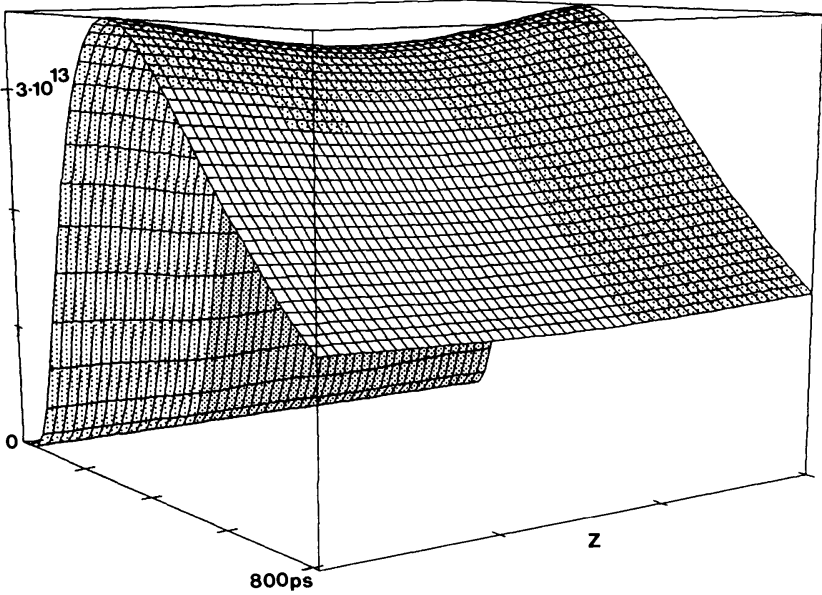

(c)

Bovine brain, $\quad(64,000 \mathrm{~m} 2 / \mathrm{s}, 42 \mathrm{ps})$ Lines @ $10 \mathrm{~mm}$ depth (0-129ps)

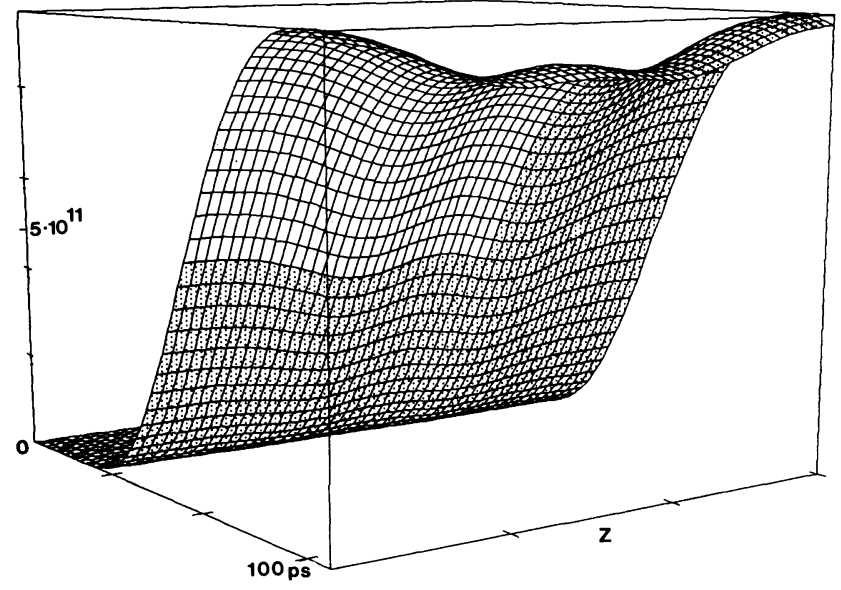

(e)
Bovine brain, 650nm (64,000m2/s:424ps) lines @ $10 \mathrm{~mm}$ depth (0-407ps)

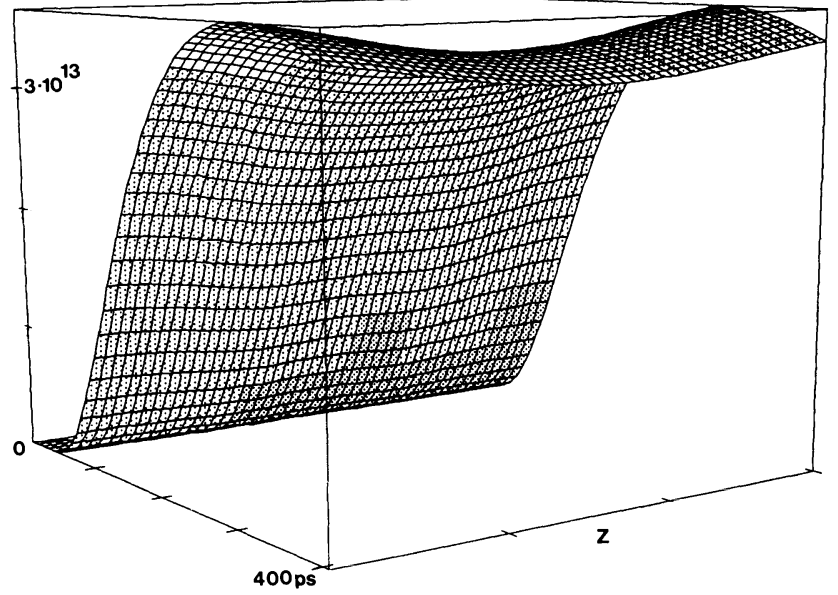

(b)

Bovine brain, $650 \mathrm{~nm}$ (64,000m2/s,424ps) Lines @ $10 \mathrm{~mm}$ depth (0-129ps)

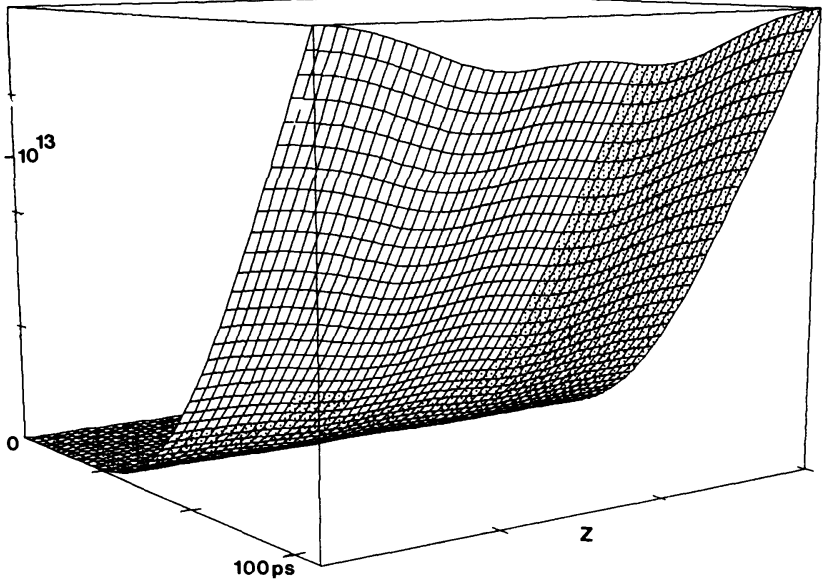

(d)

Scatter as bovine brain,Loss as sea water, (64,000m2/s,10ns) (0-1563ps)

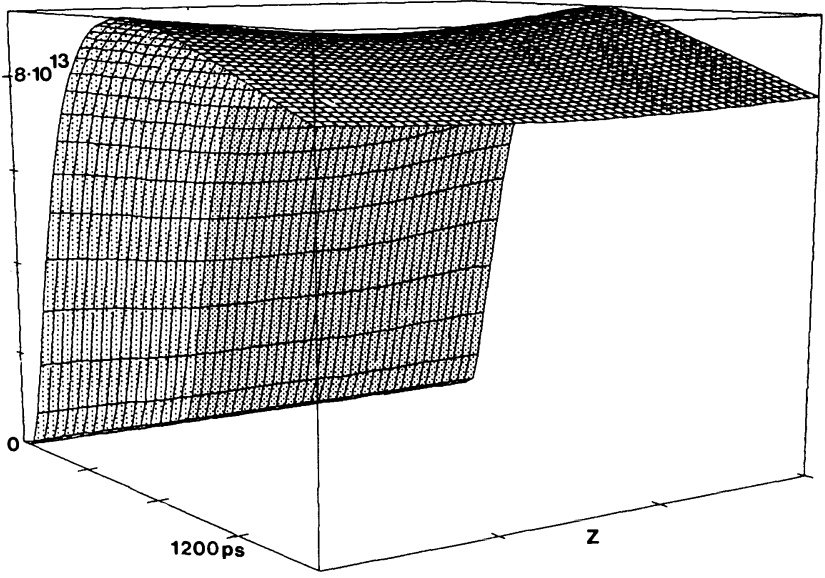

(f)

Fig. 6 Optical fluence rate at the tissue surface in $\mathrm{W} / \mathrm{m}^{2}$ [vertical after release of an optical Dirac pulse of $\left.10,000 \mathrm{~J} / \mathrm{m}^{2}\left(1 \mathrm{~J} / \mathrm{cm}^{2}\right)\right]$ in the layer embedded into the tissue. Horizontal axes describe time (picoseconds) release of the pulse and the position of two totally absorbing 1-mm lines located in the horizontal plane normal to the $z$ axis. 
when optical wavelengths corresponding to maximum penetration are used. This restriction can be improved either by selecting an optical wavelength with higher loss or by using time- or frequency-resolved techniques. The technique of enhancing the optical loss has its limitations because the relevant signal attenuation also is increased, whereas the practical applicability of time-resolved techniques is limited by the availability of inexpensive fast optical equipment. The relevant time and frequency scales are, respectively, 10 to $100 \mathrm{ps}$ and 1 to $10 \mathrm{GHz}$ for imaging of 10 - to 30-mm-deep structures. However, the progress of high-speed laser diodes and solid state detectors over the last decade indicates that optical techniques might have significant future applications for in vivo spectroscopy and imaging of tissue structures.

\section{Appendix}

The optical diffusion equation can be expressed ${ }^{6}$

$X \nabla^{2} \varphi-\frac{\partial \varphi}{\partial t}-\frac{\varphi}{\tau}=-q c$

where $\varphi$ is the optical fluence rate, $t$ is time, $q$ is the diffuse photon source density, and $c$ is the velocity of light in the medium. The optical diffusivity $X$ and the absorption relaxation time $\tau$ can be expressed ${ }^{15}$

$X=\frac{c_{0}}{3[\sigma(1-g)+\beta] n}=\frac{c_{0}}{3\left(\sigma_{\mathrm{eff}}+\beta\right) n}$,

$\tau=\frac{n}{c_{0} \beta}$,

where $\sigma$ and $g$ are, respectively, the scattering coefficient and the average cosine of the scattering angle. The parameter $\sigma_{\text {eff }}=\sigma(1-g)$ is the effective scattering coefficient, $\beta$ is the absorption coefficient, and $c_{0}$ and $n$ are, respectively, the velocity of light in vacuum and the index of refraction of the medium.

Harmonic solutions of the diffusion equation can be written in the form

$\varphi \propto \exp \left[i\left(\omega t-k_{i} x\right)\right] \exp \left(-k_{r} x\right)$,

where $k_{i}$ and $k_{r}$, respectively, are the angular repetency and the attenuation coefficient. The angular frequency is $\omega$, and $x$ is the distance in space. The relation between these quantities follows from substitution of Eq. (12) in Eq. (10) ${ }^{6}$ :

$k_{r}=\frac{1}{(2 X \tau)^{1 / 2}}\left\{\left[1+(\omega \tau)^{2}\right]^{1 / 2}+1\right\}^{1 / 2}$,

$k_{i}=\frac{1}{(2 X \tau)^{1 / 2}}\left\{\left[1+(\omega \tau)^{2}\right]^{1 / 2}-1\right\}^{1 / 2}$,

The 1-D time dependent can be found by expressing the spatial dependence of the solutions in the form

$\varphi \propto \exp (-k x)$.

The Laplace transform of the homogeneous diffusion equation can thus be expressed [Eqs. (10) and (14)] $\varphi(x, s)\left(X k^{2}-s-\frac{1}{\tau}\right)=0$,

where

$k= \pm\left[\frac{1}{X}\left(s+\frac{1}{\tau}\right)\right]^{1 / 2}$

The boundary condition at a partially transmitting radiative surface can be expressed

$\frac{X}{c} \frac{\partial \varphi}{\partial x}=A \varphi$,

where $A$ is a photon flux transfer coefficient at the surface. This quantity, which is a function of the properties of the tissue and of the surrounding medium, ranges from zero to infinity, respectively, for the totally reflecting and the totally transmitting boundary.

A finite amount of optical energy released in a layer parallel to the surface at a depth of $x=\xi$ gives the following:

$\frac{X}{c} \frac{\partial \varphi_{1}}{\partial x}-\frac{X}{c} \frac{\partial \varphi_{2}}{\partial x}=Q_{\mathrm{pl}}$,

$\varphi_{1}=\varphi_{2}$,

where $\varphi_{1}$ and $\varphi_{2}$, respectively, are the Laplace transforms of the fluence rates at $x=\xi-$ and $x=\xi+$. The quantity $Q_{\mathrm{pl}}$ is energy density released per unit area in the layer at time $t=0$.

The Laplace transform of the solutions can be expressed in the form [Eq. (14)]

$\varphi(x, s)=C_{1} \exp (k x)+C_{2} \exp (-k x) \quad$ for $0<x<\xi$,

$\varphi(x, s)=C_{3} \exp (-k x) \quad$ for $x>\xi$,

where $k$ is the positive root of Eq. (16). The constants $C_{1}$, $C_{2}$, and $C_{3}$ follow from the boundary conditions given in Eqs. (17) and (18). The solution can then be expressed in the form

$$
\begin{aligned}
\varphi(x, s)= & \frac{c Q_{\mathrm{pl}}}{2 X}\left(\frac{1}{k} \exp (-k|\xi-x|)+\frac{1}{k} \exp [-k(\xi+x)]\right. \\
& \left.-2 \frac{c A}{X}\left\{\frac{1}{k[k+(c A / X)} \exp [-k(\xi+x)]\right\}\right) .
\end{aligned}
$$

The time-dependent solution follows from the inverse Laplace transform:

$$
\begin{aligned}
\varphi(x, t)= & \mathscr{L}^{-1} \varphi(x, s)= \\
& \left(\frac{1}{2(\pi X t)^{1 / 2}}\left\{\exp \left[-\frac{(x-\xi)^{2}}{4 X t}\right]+\exp \left[-\frac{(x+\xi)^{2}}{4 X t}\right]\right\}\right. \\
& -h \exp \left[h(x+\xi)+h^{2} X t\right] \\
& \left.\times \operatorname{erfc}\left[\frac{x+\xi}{2(X t)^{1 / 2}}+h(X t)^{1 / 2}\right]\right)\left[c Q_{\mathrm{pl}} \exp \left(-\frac{t}{\tau}\right)\right],
\end{aligned}
$$


where $h=A c / X$ has been substituted.

The term containing the complementary error function can also be written

$$
\begin{gathered}
-h \exp \left[h(x+\xi)+h^{2} X t\right] \operatorname{erfc}\left[\frac{x+\xi}{2(X t)^{1 / 2}}+h(X t)^{1 / 2}\right]= \\
-\frac{h}{(\pi X t)^{1 / 2}} \int_{0}^{\infty} \exp (-h \alpha) \exp \left[-\frac{(x+\xi+\alpha)^{2}}{4 X t}\right] \mathrm{d} \alpha
\end{gathered}
$$

The fluence rate due to the release of a uniform energy density $Q_{c y l}$ per unit length of an infinite thin line parallel to the surface at time $t=0$ can correspondingly be expressed ${ }^{16}$

$$
\begin{aligned}
\varphi(x, z, t)= & \left\{\exp \left[-\frac{(x-\xi)^{2}}{4 X t}\right]+\exp \left[-\frac{(x+\xi)^{2}}{4 X t}\right]\right. \\
& \left.-\int_{0}^{\infty} 2 h \exp (-h \alpha) \exp \left[-\frac{(x+\xi+\alpha)^{2}}{4 X t}\right] \mathrm{d} \alpha\right\} \\
& \times \exp \left[-\frac{(z-\zeta)^{2}}{4 X t}\right] \frac{1}{4 \pi X t} c Q_{\mathrm{cyl}} \exp \left(-\frac{t}{\tau}\right),
\end{aligned}
$$

where the line is oriented parallel to the $\mathrm{y}$-axis and goes through the point given by $(x=\xi, z=\zeta)$.

\section{Acknowledgments}

This work was performed with support from The George Hewitt Foundation for Medical Research, the Office of Naval Research grant \#N00014-91-C-0134, and the Department of Energy grant \#DE-FG03-91 ER61227. The authors wish to thank Khai $\mathrm{Vu}$, Eric Cho, Matthew McAdams, and Joseph Ahdoot for their contributions.

\section{References}

1. S. Ertefai and A. E. Profio, "Spectral transmittance and contrast in breast diaphanography," Med. Phys. 12, 393-400 (1985).

2. G. A. Navarro and A. E. Profio, "Contrast in diaphanography of the breast," Med. Phys. 15, 181-187 (1988).

3. S. Andersson-Engels, R. Berg, S. Svanberg, and O. Jarlman, "Timeresolved transillumination for medical diagnostics," Opt. Lett. 15, 1179-1181 (1990).

4. M. S. Patterson, B. Chance, and B. C. Wilson, "Time-resolved reflectance and transmittance for the non-invasive measurement of tissue optical properties,"' Appl. Opt. 28, 2331-2336 (1989).

5. K. M. Yoo, Q. Xing, and R. R. Alfano, "Imaging objects hidden in highly scattering media using femtosecond second-harmonic-generation cross-correlation time gating," Opt. Lett. 16, 1019-1021 (1991).

6. L. O. Svaasand and B. J. Tromberg, "On the properties of optical waves in turbid media," in Future Trends of Biomedical Applications of Lasers, Proc. SPIE 1525, 41-50 (1991).

7. B. J. Tromberg, L. O. Svaasand, T. Tsay, R. C. Haskell, and M. W. Berns, "Optical property measurement in turbid media using frequency domain photon migration," in Future Trends of Biomedical Applications of Lasers, Proc. SPIE 1525, 52-58 (1991).

8. E. M. Sevick, B. Chance, J. Leigh, S. Nioka, and M. Maris, "Quantification of time and frequency-resolved optical spectra for the determination of tissue oxygenation," Anal. Biochem. 195, 330-351 (1991)

9. A. E. Profio, "Light transport in tissue," Appl. Opt. 28, 2216-2222 (1989).

10. K. M. Yoo, F. Liu, and R. R. Alfano, "Imaging through a scattering wall using absorption," Opt. Lett. 16, 1068-1070 (1991).

11. J. C. Hebden, R. A. Kruger, and K. S. Wong, "Time resolved imaging through a highly scattering medium," Appl. Opt. 30, 788-794 (1991).
12. J. Fishkin, E. Gratton, M. J. vandeVen, and W. W. Mantulin, "Diffusion of intensity modulated near-infrared light in turbid media," in Time Resolved Spectroscopy and Imaging of Tissues, Proc. SPIE 1431, 122-135 (1991)

13. "Quantities and units of light and related electromagnetic radiations,' International Standard ISO 31/6 (1980)

14. B. J. Tromberg, L. O. Svaasand, T. T. Tsay, and C. H. Haskell, "Properties of photon density waves in multiple-scattering media," Appl. Opt. (in press).

15. A. Ishimaru, "Diffusion of light in turbid materials," Appl. Opt. 28, 2210-2215 (1989)

16. H. S. Carslaw and J. C. Jaeger, Conduction of Heat in Solids, Oxford Science Publication, Oxford, pp. 371 (1959).

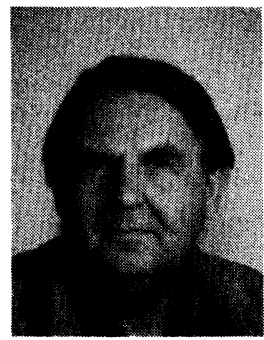

Lars O. Svaasand received the MS degree in electrical engineering and the PhD degree in physical electronics in 1961 and 1974, respectively, from the Nonwegian Institute of Technology, University of Trondheim, Norway. He has been a professor of physical electronics at the Division of Physical Electronics, Norwegian Institute of Technology, since 1982, and he served as chairman of the Department of Electrical Engineering and Computer Science at the Institute from 1984 to 1987 . He has been a visiting professor at the University of California/Santa Barbara, the University of Southern California School of Medicine, and at the University of California/ Irvine. He is a member of the American Physical Society, American Society for Laser Medicine and Surgery, American Society for Photobiology, The New York Academy of Sciences, SPIE, The Norwegian Academy of Technical Sciences, and The Royal Norwegian Society of Sciences. He is also a coeditor of the Journal Lasers in Medical Science.

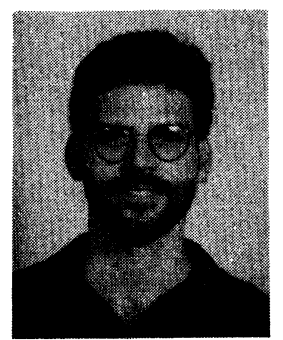

Bruce J. Tromberg is an assistant professor with joint appointments in the departments of Surgery and Physiology-Biophysics at the Beckman Laser Institute and Medical Clinic, University of California/Irvine. He completed his undergraduate studies at Vanderbilt University and was subsequently awarded a Department of Energy Predoctoral Fellowship at Oak Ridge National Laboratory and the University of Tennessee. In 1988 he received a PhD in chemistry, specializing in optical spectroscopy and fiber sensors, and began a Hewitt Foundation Postdoctoral Fellowship at the Beckman Laser Institute. Dr. Tromberg's research interests include the development of noninvasive optical techniques for monitoring physiology in single cells and tissues.

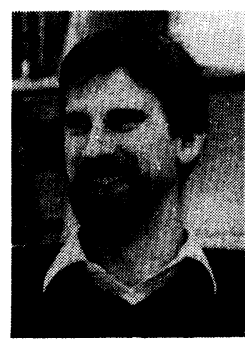

Richard C. Haskell is an associate professor of physics at Harvey Mudd College in California. He received his $\mathrm{PhD}$ in nuclear physics from Johns Hopkins University in 1972, after which he devoted six years to laser light scattering studies of skeletal muscle in the Biophysics Department at Johns Hopkins. His current interests in biophysics focus on laser probes of membrane dynamics and laser diagnostics of tissue. Haskell is a member of SPIE, the American Physical Society, and the Biophysical Society. 


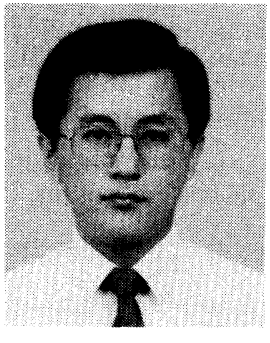

Tsong-Tseh Tsay attended the National Cheng Kung University, Taiwan, from 1976 through 1980, receiving a BS degree in physics. He was a graduate student at the University of North Carolina at Chapel Hill, receiving the $\mathrm{MS}$ and $\mathrm{PhD}$ degrees in biomedical engineering in 1986 and 1990 , respectively. Since 1990, he has held a George E. Hewitt Foundation research fellowship position at Beckman Laser Institute and Medical Clinic at the University of $\mathrm{Cal}-$ ifornia/Irvine. His current research interests include photon migration in tissue, optical spectroscopy, and imaging for biomedical applications.

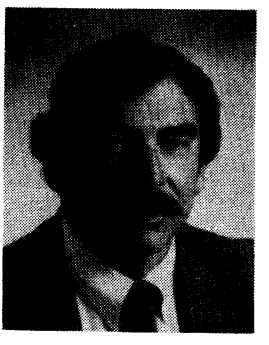

Michael W. Berns, the Arnold and Mabel Beckman professor, is president, director, and cofounder of the Beckman Laser Institute and Medical Clinic at the University of California/Irvine. He is also a professor of surgery, cell biology, radiology, and ophthalmology. Dr. Berns earned his bachelor's, master's, and doctorate degrees from Cornell University, Ithaca, New York, where he specialized in genetics, cell biology, and developmental biology. His basic research interests are focused on studies of cell genetics, cellular motility and cell movement, and the development of lasers and computers for biomedical studies. His clinical research involves the use of lasers in cancer, cardiovascular surgery, and vision. 